\title{
DESVIOS FONOLÓGICOS NA EDUCAÇÃO INFANTIL
}

\section{Phonological deviations in elementary school}

\author{
Djakelânia Alves Nunes ${ }^{(1)}$, Luzia Miscow da Cruz Payão ${ }^{(2)}$, Ranilde Cristiane Cavalcante Costa ${ }^{(3)}$
}

\section{RESUMO}

Tema: desvio fonológico na Educação Infantil. Procedimentos: analisar a intervenção fonoaudiológica baseada na hierarquia dos traços distintivos e na consciência fonológica em uma população de crianças com desvios fonológicos, estudantes da Educação Infantil. Resultados: com a análise dos dados, verificou-se maior predominância de processos de simplificação de líquidas, simplificação de encontros consonantais e simplificação da consoante final. Ocorreu a intervenção fonoaudiológica, na qual apenas uma criança não conseguiu superar as dificuldades encontradas anteriormente no processo de organização fonológica, apresentando ainda ausência de traço distintivo no sistema. Conclusão: a intervenção fonoaudiológica fundamentada na hierarquia dos traços distintivos e na consciência fonológica ampliou o inventário fonético e o sistema fonológico dessas crianças, melhorando o contraste entre os fonemas e o desempenho das habilidades de manipulação dos sons da fala, consequentemente, proporcionando a inteligibilidade de fala.

DESCRITORES: Desenvolvimento da Linguagem; Fonética; Fonoterapia

\section{INTRODUÇÃO}

Durante a aquisição fonológica, a criança adquire sons simples, e no decorrer dos anos há uma expansão desse sistema fonológico, adquirindo os sons mais complexos. Essa aquisição do sistema de sons obedece à hierarquia dos traços distintivos e as leis implicacionais entre eles ${ }^{1-3}$.

De acordo com a hierarquia preconizada na geometria de traços, esses traços distintivos delimitam as características acústicas e articulatórias que constituem os fonemas, como, por exemplo, a sonoridade, o ponto e modo de articulação sobrepostos, os quais determinam o som que é percebido quando se fala ${ }^{2,4}$.

À medida que a criança desenvolve o reconhecimento, a discriminação e a memória auditiva dos sons da fala origina-se a consciência fonológica,

(1) Aluna do Curso de Graduação em Fonoaudiologia da Universidade Estadual de Ciências da Saúde de Alagoas.

(2) Fonoaudióloga; Professora Assistente da Faculdade de Fonoaudiologia de Alagoas da Universidade Estadual de Ciências da Saúde de Alagoas, UNCISAL, Maceió, AL, Brasil; Mestre em Linguística.

(3) Fonoaudióloga; Professora Auxiliar da Faculdade de Fonoaudiologia de Alagoas da Universidade Estadual de Ciências da Saúde de Alagoas, UNCISAL, Maceió, AL, Brasil; Especialista em Docência no Ensino Superior.

Conflito de interesses: inexistente manifestada por habilidades de manipulação consciente dos sons das palavras ${ }^{1}$.

Nos desvios fonológicos as crianças apresentam dificuldades específicas na organização fonológica necessária para o aprendizado da linguagem oral. A maioria delas apresenta ininteligibilidade de fala, tornando a mensagem ambígua para seu interlocutor. Essas crianças se desenvolvem de forma semelhante às outras, portanto, sem dificuldades de aprendizagem geral, na ausência de déficits intelectual e auditivo ou fatores etiológicos conhecidos ${ }^{5,6}$.

A terapia baseia-se na investigação dos processos fonológicos presentes, tendo como objetivo facilitar o desenvolvimento de um sistema fonológico inteligível, por meio da supressão de processos fonológicos durante o tratamento, assemelhandose ao sistema alvo do adulto ${ }^{5,6}$.

A presente pesquisa tem o objetivo de analisar a intervenção fonoaudiológica baseada na hierarquia dos traços distintivos e na consciência fonológica em um grupo de quatro crianças com desvio fonológico, na faixa etária entre 04 e 07 anos, estudantes da Educação Infantil, visto que este estudo possibilita o início de uma pesquisa fonoaudiológica inédita na área dos distúrbios da comunicação humana da população infantil alagoana, fornecendo informações locais quanto às características linguísticas 
e as potencialidades de expressão verbal. Além disso, viabiliza a avaliação e tratamento fonoaudiológico, área da saúde específica para a prevenção e reabilitação dos distúrbios da fala e linguagem.

\section{APRESENTAÇÃO DO CASO}

A pesquisa foi realizada na Unidade de Tratamento em Fonoaudiologia Prof. Jurandir Bóia Rocha (UTFONO), vinculado à Universidade Estadual de Ciências da Saúde de Alagoas - UNCISAL, Faculdade de Fonoaudiologia de Alagoas.

Foram avaliadas quatro crianças na faixa etária de 04 a 07 anos de idade, estudantes da Educação Infantil da rede pública de ensino, no período de agosto de 2006 a agosto de 2007. Os critérios utilizados na escolha desses sujeitos foram crianças apresentando alterações na fala, sem nenhum fator orgânico e etiológico que comprometesse a linguagem.

Cada criança foi avaliada individualmente pelas fonoaudiólogas participantes desta pesquisa, sendo que a avaliação consistiu de etapas.

Na primeira etapa foi realizada anamnese com os genitores, para se obter o perfil do desenvolvimento cognitivo, motor e linguístico de cada criança.

$\mathrm{Na}$ segunda etapa, houve a avaliação das crianças, sendo realizada coleta de dados de fala espontânea, a partir de atividades lúdicas, como jogos, brinquedos, para verificar a presença de alterações nos fonemas, pois a fala espontânea proporciona uma amostra típica da criança e possibilita a observação dos processos fonológicos. Porém, uma desvantagem encontrada nesse método é fazer a criança com alterações de fala evitar palavras que contenham os sons mais difíceis para ela, dificultando o início da conversação.

O método de coleta foi realizado por meio de gravação em áudio, utilizando um aparelho MD (Mini-disc Recorder MZ-R700-Sony), microfone Sony unidirecional F-V120 e MP3 Sony 512 MB.

$\mathrm{Na}$ etapa seguinte foram avaliadas minuciosamente as crianças que apresentaram alterações de fala. Aplicaram-se o Teste de Linguagem Infantil, constando de prova de imitação e nomeação, as quais possibilitam uma amostra significativa da fala da criança com todos os sons da língua em diferentes situações silábicas ${ }^{7}$.

Os sujeitos da pesquisa também foram submetidos a processo avaliativo específico do sistema estomatognático, visto que os órgãos fonoarticulatórios devem estar em equilíbrio para desempenhar suas funções, que são essenciais para a aquisição e desenvolvimento de linguagem. A via aérea superior foi avaliada por um médico otorrinolaringologista. A avaliação audiológica constou de audiometria tonal, audiometria vocal, imitanciometria e processamento auditivo. Essas avaliações foram efetuadas a fim de detectar alteração auditiva que pudesse comprometer a percepção auditiva entre os fonemas e assim, a consciência fonológica. Todos os procedimentos foram realizados no serviço de atendimento fonoaudiológico da UNCISAL.

Uma vez que os dados foram coletados e registrados, foram realizadas a transcrição fonética e a análise dos processos fonológicos encontrados, possibilitando às terapeutas identificarem a particularidade de cada criança sobre o seu sistema fonológico.

Em seguida, foi executado o planejamento terapêutico, no qual as palavras alvo selecionadas basearam-se nos padrões fonológicos de cada criança. Os sons alvo faziam parte do vocabulário das crianças, facilitando o desempenho nas atividades propostas durante a terapia.

A terapia fonoaudiológica era realizada semanalmente, com duração de 30 minutos cada. Houve a participação dos pais no processo terapêutico, sendo orientados a trabalhar com as crianças em casa, usando as palavras-alvo de forma lúdica, por meio da utilização de jogos que foram confeccionados pela terapeuta e entregues à família, incentivando a generalização dos fonemas adquiridos para a linguagem espontânea.

Esse trabalho foi aprovado pelo Comitê de Ética em Pesquisa da Universidade Estadual de Ciências da Saúde de Alagoas - UNCISAL, protocolo $n^{\circ}$ 514, conforme a Resolução 196/96 do Conselho Nacional de Saúde. Todos os responsáveis pelos sujeitos consentiram na realização desta pesquisa e na divulgação de seus resultados por meio da assinatura do Termo de Consentimento Livre e Esclarecido.

\section{RESULTADOS}

Os sujeitos da presente pesquisa apresentaram predominância dos seguintes processos fonológicos: simplificação de líquidas, com ausência do traço [+ contínuo], simplificação de encontros consonantais, com omissão do fonema-alvo $/ \Gamma /$, a vibrante simples, em estrutura silábica complexa e na simplificação da consoante final.

Ocorreu a intervenção fonoaudiológica baseada na hierarquia dos traços distintivos e na ênfase de atividades que promovessem a consciência fonológica. Inicialmente foi trabalhado o fonema alvo visando à estimulação do traço distintivo comprometido e ao desenvolvimento da consciência fonológica. A terapia enfatizava a adequação dos padrões fonológicos na fala da criança, fornecendo pistas visuais, auditivas, táteis e cinestésicas, tendo como principal objetivo a generalização dos fonemas. 
No decorrer das sessões esses sujeitos expandiram seu inventário fonético e sistema fonológico, ampliando a contrastividade entre os fonemas e/ou melhora de desempenho das habilidades de manipulação dos sons da fala, consequentemente, proporcionando a inteligibilidade de fala.

Houve uma evolução significativa nos sujeitos acompanhados, sendo observado que apenas um não conseguiu superar as dificuldades encontradas anteriormente no processo de organização fonológica, apresentando ainda ausência de traço distintivo [+contínuo] na classe das líquidas.

A Tabela 1 apresenta os processos fonológicos encontrados predominantes nos sujeitos desta pesquisa.
Na Tabela 2 encontram-se amostras que relacionam o processo fonológico com o traço ausente correspondente, o qual acarreta a simplificação da fala.

Quanto à avaliação otorrinolaringológica dos quatros sujeitos participantes, apenas um apresentou otite flutuante. Esta mesma criança, na avaliação audiológica, apresentou perda auditiva condutiva de grau leve; quanto às demais não apresentaram nenhuma alteração auditiva. $\mathrm{Na}$ avaliação do sistema miofuncional oral nenhuma das crianças apresentou comprometimento orgânico (Tabela 3).

Tabela 1 - Processos fonológicos predominantes nos sujeitos

\begin{tabular}{|c|c|c|c|}
\hline Sujeitos & Simplificação de Líquidas & $\begin{array}{l}\text { Simplificação de } \\
\text { Encontro Consonantal }\end{array}$ & Eliminação da Coda Final \\
\hline Sujeito 1 & 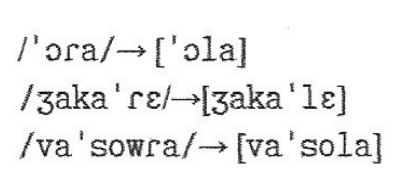 & $\begin{array}{l}/ \text { 'brîkku/ } \rightarrow \text { ['biku] } \\
/ \text { 'pregu/ } \rightarrow[\text { 'pegu }] \\
/ \text { 'grosu/ } \rightarrow \text { ['gosu }]\end{array}$ & $\begin{array}{l}/ \text { dois } / \rightarrow[\text { ['doi }] \\
/ \text { 'lapis } / \rightarrow[\text { [']api }] \\
/{ }^{\prime} \text { krus } / \rightarrow[\text { 'kuj] }\end{array}$ \\
\hline Sujeito 2 & 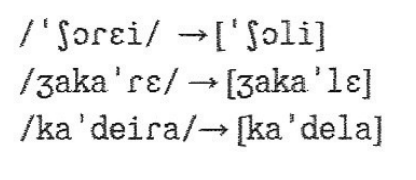 & $\begin{array}{l}/ \text { 'brãku/ } \rightarrow[\text { 'bãku }] \\
/ \text { 'kravu/ } \rightarrow[\text { 'kavu }] \\
/ \text { 'brasu/ } \rightarrow[\text { 'basu }]\end{array}$ & $\begin{array}{l}/ \mathrm{d} d s / \rightarrow\left[{ }^{\prime} \mathrm{d} \varepsilon j\right] \\
/ \mathrm{na}^{\prime} \mathrm{ris} / \rightarrow\left[\mathrm{na}^{\prime} \mathrm{Ii}\right] \\
/ \text { 'krus/ } \rightarrow\left[{ }^{\prime} \mathrm{kuj}\right]\end{array}$ \\
\hline Sujeito 3 & $\begin{array}{l}/ \text { ama'relu/ } \rightarrow \text { [ama'lelu }] \\
/ 3 i^{\prime} r a f a / \rightarrow\left[3 i^{\prime} l a f a\right] \\
/ \text { te'zowra/ } \rightarrow[\text { ti'zowla }]\end{array}$ & $\begin{array}{l}/ \text { 'kwatru/ } \rightarrow \text { ['kwatu }] \\
/ \text { 'droga/ } \rightarrow[\text { 'doga }] \\
/ \text { 'livru/ } \rightarrow \text { ['livu }]\end{array}$ & $\begin{array}{l}/ \text { tres } / \rightarrow \text { ['tej] } \\
/ \text { 'lapis } / \rightarrow \text { ['lapi] } \\
/ \text { 'krus } / \rightarrow[\text { ku'i] }\end{array}$ \\
\hline Sujeito 4 & & $\begin{array}{l}/ \text { floh/ } \rightarrow[\text { ['fo] } \\
/ \text { tra'vesa/ } \rightarrow[\text { ta'vesa }] \\
/ \text { 'plãta/ } \rightarrow[\text { 'pãta }]\end{array}$ & \\
\hline
\end{tabular}

Tabela 2 - Correlações entre processos fonológicos e os traços distintivos ausentes

\begin{tabular}{|c|c|c|c|}
\hline $\begin{array}{l}\text { Traços } \\
\text { Distintivos }\end{array}$ & Simplificação de Líquidas & $\begin{array}{l}\text { Simplificação de } \\
\text { Encontro Consonantal }\end{array}$ & Eliminação da Coda Final \\
\hline $\begin{array}{l}\text { Traço } \\
\text { [+/-contínuo] }\end{array}$ & $\begin{aligned} \mid r / & \rightarrow[1] \\
{[+ \text { continuo }] } & \rightarrow[- \text { continuo }]\end{aligned}$ & $\begin{aligned}|r| & \rightarrow \varnothing \\
{[+ \text { contínuo }] } & \rightarrow \varnothing\end{aligned}$ & $\begin{array}{c}/ s / \rightarrow \emptyset \\
{[- \text { soante/ } / \text { contínuo }] \rightarrow \emptyset}\end{array}$ \\
\hline & & $\begin{array}{c}/ 1 / \rightarrow \emptyset \\
{[- \text { continuo }] \rightarrow \emptyset}\end{array}$ & \\
\hline
\end{tabular}


Tabela 3 - Resultados das avaliações otorrinolaringológica e audiológica

\begin{tabular}{ccc}
\hline Sujeitos & Avaliação Audiológica & Avaliação Otorrinolaringológica \\
\hline Sujeito 1 & Normal & Normal \\
Sujeito 2 & Normal & Normal \\
Sujeito 3 & Perda Auditiva Condutiva & Otite Flutuante \\
Sujeito 4 & Normal & Normal \\
\hline
\end{tabular}

\section{DISCUSSÃO}

A partir da análise dos resultados, tornou-se possível o estabelecimento de uma série de informações a respeito da abordagem terapêutica em crianças com desvios fonológicos.

Todas as crianças deste estudo mostraram desempenho satisfatório nas atividades fundamentadas no desenvolvimento da consciência fonológica, que enfatizavam a percepção do traço distintivo ausente no sistema de cada criança. Esses resultados podem ser explicados pela sistemática de apresentação das palavras-alvo utilizadas nas terapias. Nessas palavras havia os fonemas que estavam comprometidos no sistema fonológico das crianças $4,8,9$.

As atividades de consciência fonológica solicitavam às crianças tarefas envolvendo semelhanças, diferenças e ordem dos sons nas palavras, proporcionando uma compreensão maior do seu sistema fonológico e desenvolvimento das habilidades metafonológicas, importantes para o processo de alfabetização ${ }^{10-14}$.

No decorrer da terapia fonoaudiológica foi observado que a evolução do sistema fonológico está relacionada com o desempenho da consciência fonológica. À medida que eles melhoravam o desempenho dessa habilidade, aumentavam seus inventários fonológicos, o que proporcionava a inteligibilidade de fala ${ }^{11-13,15,16}$.

Dentre os processos fonológicos predominantes encontram-se a omissão de líquida vibrante em encontro consonantal e a substituição desta pela líquida lateral ${ }^{3,6,16}$. A presença desses processos fonológicos na linguagem oral das crianças deste estudo corrobora as pesquisas na área de aquisição fonológica, nas quais as autoras salientam que as líquidas são de domínio mais tardio na aquisição ${ }^{6,17}$.

Esses resultados também coincidem com estudos sobre a aquisição do onset complexo, que é adquirido num primeiro momento com a líquida vibrante simples $/ \Gamma /$, sendo esse encontro con- sonantal mais frequente na língua portuguesa, ao contrário dos encontros consonantais com a líquida lateral ///, cuja frequência é menor na língua ${ }^{14,18}$.

Quanto à aquisição da coda, a consoante final de sílaba no sistema fonológico dessas crianças, observou-se um domínio tardio da fricativa em coda final, ocorrendo, desta forma, a omissão desse som nessa estrutura silábica. Esse resultado não condiz com outras pesquisas, que apontam essa posição final mais favorável ao surgimento da fricativa do que em coda medial na palavra ${ }^{15,19}$

A superação da ausência dos traços distintivos pode ser explicada pela hierarquia na geometria de traços, facilitando o estabelecimento das metas para as mudanças fonológicas que proporcionarão a inteligibilidade da fala em crianças com desvios fonológicos. À medida que se desenvolve o sistema fonológico, a criança vai especificando o traço que não estava presente inicialmente, superando, assim, os processos de substituição de fonema e de simplificação de estrutura silábica ${ }^{2,16,20}$.

É importante ressaltar que um dos fatores que influenciaram na evolução do quadro clínico das crianças foi a participação da família em algumas sessões terapêuticas, promovendo tanto o interesse como a motivação na terapia. A fala da criança com desvio fonológico por não ser entendida muitas vezes pelos adultos dificulta a interação entre eles, prejudicando assim, o desenvolvimento da linguagem. Foi necessário confeccionar jogos lúdicos associados ao fonema-alvo para incentivar e estimular a consciência fonológica também no ambiente familiar ${ }^{3-5}$.

\section{CONCLUSÃO}

A intervenção fonoaudiológica, fundamentada na hierarquia dos traços distintivos e na consciência fonológica, ampliou o inventário fonético e o sistema fonológico das crianças, melhorando o contraste entre os fonemas e o desempenho das habilidades de manipulação dos sons da fala, consequentemente, proporcionando a sua inteligibilidade. 


\section{ABSTRACT}

Background: phonological deviations in elementary school. Procedures: analyzing the phonetic approach based on the ranking of distinctive features and on phonological awareness in a population of children with phonological deviations, students of elementary school. Results: with the analysis of the information, we noted a great predominance simplification of liquids process, simplification of consonant cluster and simplification of final coda. Speech therapy took place, in which a child did not just get to overcome the difficulties found previously in the process of phonological organization, still presenting absence of distinctive trace in the system. Conclusion: the phonetic approach to phonological deviation based the ranking of distinctive features and on phonological awareness expanded the phonetic inventory and those children's phonological system, improving the recruiting between the phonemes and the performance of the abilities concerning manipulation speech sounds, consequently providing speech intelligibility.

KEYWORDS: Language Development; Phonetics; Speech Therapy

\section{REFERÊNCIAS}

1. Schuele CM, Boudreau D. Phonological awareness intervention: beyond the basics. Lang Speech Hear Serv Schools. 2008; 39(1):3-20. http:// dx.doi.org/10.1044/0161-1461(2008/002)

2. Clements GN, Hume $\mathrm{E}$. The internal organization of speech sounds. In: Goldsmith JA, organizador. The handbook of phonological theory. Cambridge: Blackwell; 1995. p. 245-306.

3. Galea DES, Wertzner HF. Índices de fala em crianças em desenvolvimento fonológico típico. Pediatr. 2005; 27(3):172-80.

4. Santos-Carvalho B, Mota HB, Keske-Soares $M$. Teste de figuras para discriminação fonêmica: uma proposta. Rev Soc Bras Fonoaudiol. 2008; 13(3):207-17.

5. Mota HB, Bagetti T, Keske-Soares M, Pereira LF. A generalização baseada nas relações implicacionais em sujeitos submetidos à terapia fonoaudiológica. Pró-Fono. 2005; 17(1):99-110.

6. Payão LMC. Desvios fonológicos em crianças da Educação Infantil: uma análise a partir da hierarquia dos traços distintivos. In: Denilda Moura, organizador. Os desafios da língua: pesquisas em língua falada e escrita. Vol. 1. Maceió: Edufal; 2008. p. 341-3.

7. Wertzner HF. Fonologia. In: Andrade CRF, BefiLopes DM, Fernades FDN, Wertzner HF. ABFW: Teste de Linguagem Infantil nas áreas de fonologia, vocábulo, fluência e pragmática. São Paulo: PróFono; 2004.p.5-16.

8. Rodríguez VMA, León SL. Contributions to the problems of generalization in the phonological intervention: a clinical approach. Rev. CEFAC. 2007; 9(1):21-31. http://dx.doi.org/10.1590/S151618462007000100004
9. Zuanetti PA, Schneck APC, Manfredi AKS. Consciência fonológica e desempenho escolar. Rev. CEFAC. 2008; 10(2):168-74. http://dx.doi. org/10.1590/S1516-18462008000200005

10. Anthony JL, Francis DJ. Development of phonological awareness. Curr Direct Psychol Sci. 2005; 14(5):255-9.

11. Mota HB, Melo Filha MGC, Lasch SS. A consciência fonológica e o desempenho na escrita sob ditado de crianças com desvio fonológico após realização de terapia fonoaudiológica. Rev. CEFAC.2007;9(4):477-82.http://dx.doi.org/10.1590/ S1516-18462007000400007

12. Paes CTS, Pessoa ACRG. Habilidades fonológicas em crianças não alfabetizadas e alfabetizadas. Rev. CEFAC. 2005; 7(2):149-57.

13. Santamaría VL, Leitão PB, Assencio-Ferreira VJ. A consciência fonológica no processo de alfabetização. Rev. CEFAC. 2004; 6(3):237-41.

14. Paula GR, Mota HB, Keske-Soares M. A terapia em consciência fonológica no processo de alfabetização. Pró-Fono. 2005; 17(2):175-84.

15. Tavares JG, Payão LMC. Análise da fala de uma criança com desvio fonológico: estudo de caso clínico. J Bras Fonoaudiol. 2006; 6(24):51-9.

16. Spíndola RA, Payão LMC, Bandini HHM. Abordagem fonoaudiológica em desvios fonológicos fundamentada na hierarquia dos traços distintivos e na consciência fonológica. Rev. CEFAC. 2007; 9(2):180-9. http://dx.doi.org/ S1516-18462007000200006

17. Wertzner HF, Pagan LO, Gálea DES, Papp ACCS. Características fonológicas de crianças com transtornos fonológicos com e sem histórico de otite média. Rev Soc Bras Fonoaudiol. 2007; 12(1):41-7. 
18. Ribas LP. Sobre a aquisição do onset complexo. In: Lamprecht RR. Aquisição fonológica do Português: perfil de desenvolvimento e subsídios para terapia. Porto Alegre: Artmed; 2004. p.151-64. 19. Mezzomo CL. Sobre a aquisição da coda. In: Lamprecht RR. Aquisição fonológica do Português: perfil de desenvolvimento e subsídios para terapia. Porto Alegre: Artmed; 2004. p.129-50.

20. Storkel HL, Morrisette ML. The lexicon and phonology: interactions in language acquisition. Lang Speech Hear Serv Schools. 2002; 33(1):24-37.

RECEBIDO EM: 19/05/2008

ACEITO EM: 02/12/2009

Endereço para correspondência:

Djakelânia Alves Nunes

Rua Presidente Médici, 42

Arapiraca - AL

CEP: $57312-450$

E-mail: kekeu.nunes@ hotmail.com 